\title{
Analysis of Different M-Band Wavelet Filters for Face Recognition using Nearest Neighbor Classifier
}

\author{
C. Hemalatha ${ }^{1}$, E. Logashanmugam ${ }^{2}$ \\ ${ }^{1}$ St. Peter's University, Chennai, India \\ ${ }^{2}$ Department of ECE, Sathyabama University, Chennai, India
}

\begin{tabular}{|c|c|}
\hline Article Info & ABSTRACT \\
\hline Article history: & \multirow{11}{*}{$\begin{array}{l}\text { Face recognition system is one of the most interesting studied topics in } \\
\text { computer vision for past two decades. Among the other popular biometrics } \\
\text { such as the retina, fingerprint, and iris recognition systems, the face } \\
\text { recognition is capable of recognizing the uncooperative samples in a non- } \\
\text { intrusive manner. Also, it can be applied to many applications of surveillance } \\
\text { security, forensics, border control, digital entertainment where face } \\
\text { recognition is used in most. In the proposed system an automatic face } \\
\text { recognition system is discussed. The proposed recognition system is based on } \\
\text { the Dual-Tree M-Band Wavelet Transform (DTMBWT) transform algorithm } \\
\text { and features obtained by varying the different filter in the DTMBWT } \\
\text { transform. Then the different filter features are classified by means of the K- } \\
\text { Nearest Neighbor (KNN) classifier for recognizing the face correctly. The } \\
\text { implementation of the system is done by using the ORL face image database, } \\
\text { and the performance metrics are calculated. } \\
\text { Copyright } \odot 2018 \text { Institute of Advanced Engineering and Science. } \\
\text { All rights reserved. }\end{array}$} \\
\hline Received Apr 9, 2018 & \\
\hline Revised May 20, 2018 & \\
\hline Accepted Jul 11, 2018 & \\
\hline Keywords: & \\
\hline \multirow{6}{*}{$\begin{array}{l}\text { DTMBWT } \\
\text { Face recognition } \\
\text { KNN } \\
\text { M-band } \\
\text { ORL database }\end{array}$} & \\
\hline & \\
\hline & \\
\hline & \\
\hline & \\
\hline & \\
\hline \multicolumn{2}{|l|}{ Corresponding Author: } \\
\hline $\begin{array}{l}\text { C. Hemalatha, } \\
\text { St. Peter's University, } \\
\text { Chennai, India. } \\
\text { Email: hemac.18@ rediffmail.com }\end{array}$ & \\
\hline
\end{tabular}

\section{INTRODUCTION}

In recent years, the face recognition is becoming an importand and classical research topic in the field of computer vision and also in pattern recognition because of its wide variety real world applications like video surveillance system, security access systems and human-computer intelligent interaction systems, etc. But the recognition of face is not an easy task to work. Hence in this system, we are implementing an algorithm of face recognition system as explained in the forecoming sections.

\subsection{Background}

The exploration of Multi-Task Learning (MTL) for face recognition method is discussed in [1]. At first, the multi-task Convolutional Neural Network (CNN) is used for the recognition of the face in which the identity classification is the main task of pose, illumination, and expression estimation. Then a scheme of dynamic-weighting is automatically assigned for the loss of weights to each side task. Finally, a posedirected multi-task CNN is grouped by various poses to learn the pose-specific identity features present in the framework.

Illumination invariant extraction based on the estimation of illumination is used widely in alleviating the adverse effects of illumination variations. In [2], a method based on the estimation of extracting the directional illumination invariant sets from a facial image is discussed. The directional illumination is used for preserving the essential features of the face and also for reducing the adverse effects of quick light changes. Additionally, a metric of multilevel matching is used for the classification of category based on the residual matching. 
A system of face recognition based on the fuzzy linear regression discriminant projection algorithm is discussed in [3]. The algorithm is helpful for producing an energy subspace for the linear regression discriminant and to handle the variations occurred in facial images. The algorithm first computes the input samples gradual membership degree concerning its classes then the membership degree is used for building the fuzzy system. Finally, the ratio of the fuzzy is maximized for the generation of criterion function.

Face recognition technique based on a category-particular dictionary, and a projection matrix is discussed in [4]. At first, the training facts are being decomposed into the classes like the class-specific dictionary, sparse blunders matrix and non-elegance-unique dictionary. Secondly, the information's are projected onto their underlying corresponding subspace, and as well as the projection matrix between the genuine training information and the dictionary of elegance-precise found. Finally, the features of the elegance-unique dictionary are extracted with the help of Eigen face technique.

A deep face recognition system that addresses the problems occurs in an open set protocol is discussed in [5]. The method uses the angular softmax loss that actives the convolutional neural network in order to learn the features of the angular discrimination. In the geometrical perception, the angular softmax loss is imposed as the discriminative constraints on the hyper sphere. Also, the angular marginal size can be adjusted quantitatively by a parameter.

An adaptive linear discriminant regression classification algorithm for face recognition system is discussed in [6]. The algorithm is used to first characterize the various contributions of the training samples with the help of the different weights. Then the weighting information is used to calculate the reconstruction errors of within-class and between-class errors. Finally, the algorithm maximizes the ratio of the betweenclass reconstruction error over the within-class reconstruction error by means of the optimal projection matrix.

A method used for unconstrained face recognition in the wild that pushes the frontiers of the extreme pose variations is discussed in [8]. A single model of pose invariant is learned by the greater amount of training data samples that normalize the single frontal pose. The method uses the multiple pose-specific models of the rendered face images in order to tackle the pose variations explicitly. Then the deep convolutional neural network is used to learn the discriminative representations of the images.

A method of face recognition system based on the arbitrarily shaped kernels in the presence of space varying blur motion is discussed in [8]. The blurred face images are used as the model for the convex combination of the geometrically transformed instances to show that convex set of images are obtained by the non-uniformly blurring of the images. Also, a method based on the non-uniform blur robust algorithm is used to build an energy function from sparse camera trajectory with the 11-norm constraint of the space motion.

A system of face recognition method based on the Local Zernike Moments (LZM) is discussed in [9]. The method is of two schemes. At first, the phase magnitude histogram is used over the complex components of LZM. Secondly, the Local Zernike Xor Patterns (LZXP) is generated and used for encoding the phase components. For both the schemes the images are divided into the sub-regions, and then by concatenating the histograms, the feature vectors are constructed for all sub-bands.

Analysis of regression-based classification method for face recognition system is discussed in [10]. The system uses the nuclear norm regularized regression method along with the occlusion for recognition. The method integrates the error support and error detection into one model of regression.

A face recognition system, based on the method called completed local binary pattern algorithm that uses the texture features is discussed in [11]. The method is used to investigate the variants of the local binary pattern in the fields of scene, texture and events of the face image classification. A modified version of the Least Trimmed Squares (LTS) along with a genetic algorithm is discussed in [12]. The method is used modify the LTS along with genetic algorithm method. The use of the genetic algorithms is to construction of the subsets rather than the random selection of the basic subsets.

A method based on the one-dimensional hidden Markov classification model for performing the low face recognition is discussed in [13]. The method uses three steps for the extraction of the facial features as such; the calculation of the histogram of oriented gradients descriptor and the Gabor filters, then the linear discriminant analysis method is used to remove the redundant information and to reduce the feature sizes and at last the canonical correlation analysis method is used to combine the features before the classification.

A method for the recognition of the face after the plastic surgery based on the Entropy based scale invariant feature transform (EV-SIFT) method is discussed in [14]. This feature will extract the key points and the scale-space structure volume information. Since the entropy is a type of the higher order statistical feature, the uncertainty variations in the will have the least effects in it and so the features are classified by using the support vector machine classifier. 


\subsection{Problem Statement}

When developing an automatic face recognition system one may encounter few problems such as:

a) It is known that for the recognition of face, the size of the image will be quite small that acts as the disadvantages as the face recognition system that purely depends on the accurate feature localizations.

b) The problem of producing the accurate feature location is difficult in order to obtain a good face recognition performance.

\subsection{The Proposed Solution}

To overcome these problems, an automatic face recognition method based on algorithm called the DTMBWT transform from which the features are extracted by using the different features present in the transformation algorithm. Then the system is classified by using the KNN classifier scheme.

The paper organized in a way that the section 2 explaines about the methodology, the features extraction filters and the classification methods used. Then the section 3 shows discussion about the results obtained and the section 4 gives a conclusion for this automatic face recognition system.

\section{METHODOLOGY}

The proposed face recognition system is made up of two types of operations such as training and the testing phases. In the training phase, all the training face images are used for the extraction of the features. Before the extraction of features, the inputs are decomposed by using the algorithm known as Dual Tree MBand Wavelet Transform (DTMBWT) transform. Then the features are extracted from the transformed image by varying the different filter available in DTMBWT and are saved as the trained database. Then the testing phase is done by using the test image and to classify the features that are extracted from the testing set of images. To perform the action of classification the classifier algorithm used in the proposed system is the KNN classifier. The block diagram of the proposed system is shown in Figure 1.

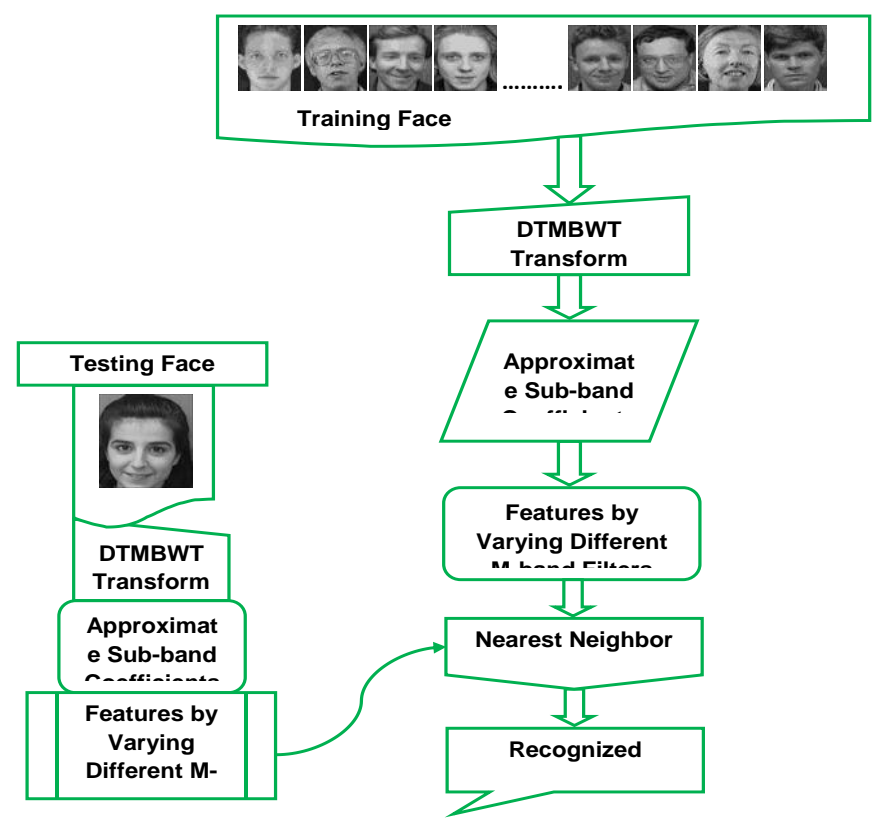

Figure 1. Block diagram for the proposed face recognition system

\subsection{Feature Extraction}

In any classification or recognition system, the feature extraction process is carried out very importantly. The features that are extracted from the input images are useful for the classification purpose as the classifier inputs. In our proposed system also the features extraction process is performed by using the algorithm known as the DTMBWT transform algorithm. As this algorithm is one of the types of wavelet transform method the features are extracted by means of the sub-band coefficients. Using the DTMBWT transform, the high and low-frequency sub-band coefficients are obtained from the input images are obtained 
and are considered as the features. The dual-tree wavelet transform was first practiced by Selesnick [15] and was improved by Chaux.

The dual-tree M-band wavelets are considered to be more selective towards the frequency domain. The DTMBWT transform is used for decomposing the given input image at pre-defined levels and helps in computing the feature vectors with the $\mathrm{M}$ band filter banks. By using this transform number of highfrequency sub-band and low-frequency sub-band coefficients are obtained that depend on the filter banks and the decomposition levels. It is illustrated as $J \times M^{2}-J+1$ here $\mathrm{J}$ is referred as the number of decomposition levels and $\mathrm{M}$ is referred as the number of filter banks. The M-band dual tree transforms consists of three steps such as; Prefiltering, M-band wavelet decomposition and Direction extraction from various sub-bands.

\subsection{M-Band Wavelet Filters}

The Wavelet transforms constitute a well-established tool that is used for image processing applications. Some of the popular extensions to the wavelet transform are the wavelet packet transforms, and the M-band wavelet transforms. There is some relationship between the filter banks and the wavelet frames under some certain conditions. In this system, we are focusing on the 1D Dual-Tree M-band Transform (DTT) filter specifications. From the available filter for the DTT, the filters like Haar band, Meyer band, Symlets band filters are explained as follows:

\subsubsection{HAAR Wavelet Filter}

The wavelet haar is said to be a sequence of square-shaped rescaled functions that together form a wavelet basis or family as discussed in [16]. The analysis of wavelet is as similar to that of the Fourier analysis that allows the target function to be represented as orthonormal basis function over an interval of time. The haar wavelets are represented as $\left({ }^{\psi}\right)$ and the scaling wavelet is represented as $\left({ }^{\phi}\right)$.

\subsubsection{MEYER Wavelet Filter}

The Meyer wavelet function can be represented as the discrete format of Dmey wavelet filter [16]. The Meyer's wavelet equation generally acts as a solvent method for solving the two-scale equation. Based on the approximation space basis, the Meyer employed the Fourier transform in order to derive the coefficients two-scale education.

\subsubsection{SYMLET Wavelet Filter}

The symlet wavelet filter defines the family of orthogonal wavelets and is also called as the least asymmetric wavelet basis [16]. The symlet wavelet function is usually defined for any positive integer $n$ whereas the wavelet function $\left({ }^{\psi}\right)$ and the scaling function $\left({ }^{\phi}\right)$ that supports the length of $2 \mathrm{n}$. The symlet can be used as the functions such as wavelet phi and discreet wavelet transforms.

Using these filters, the features are extracted from the training images and are saved as the trained database which is later used. Whereas the same set of filters are used for the testing images and are used as the inputs for the classifier that performs the recognition process.

\subsection{Classification}

The output of the proposed face recognition system mainly depends on the classification step that is carried out for the decision-making process by using an effective classifier with it. In our proposed system the decision-making process is made by using the K-Nearest Neighbor (KNN) classifier algorithm. The KNN helps in classifying the features based on the training examples in the closest feature vector.

The classification is made successfully based on the majority of the vote by its neighbors. The $\mathrm{k}$ refers to the values that are decided based on the data size used for classification. When $\mathrm{k}$ is set to $1,(\mathrm{k}=1)$, then the features are assigned to its nearest neighbor class. When the size of $\mathrm{k}$ is increased then the effects of noise is also increased, and also the boundaries between the classes are decreased. The prediction for the test samples are made by following the steps like computing the distance of test vectors along with the train vectors. To find the closest vectors of $\mathrm{k}$ and also to arrange the distance in ascending order and to choose the closest label. In this work the KNN algorithm is used with the Euclidean distance measures is used for classification. Equation 1 shows the Euclidean distance formula as:

$$
d(x, y)=\|x-y\|=\left[\sum_{i=1}^{m}\left(x_{i}-y_{i}\right)^{2}\right]^{\frac{1}{2}}
$$


Where $\mathrm{x}$ and $\mathrm{y}$ denote the Euclidean vector. Using this Euclidean distance measures the classification is done by using the feature inputs given to the classifier. The features obtained from different filters of the $\mathrm{m}$-band transform from the training and the testing samples are given as the inputs for the classifier. Then the classifier compares both the features obtained from the training and the testing process and makes the decision for the recognition of face successfully.

\section{RESULT AND DISCUSSION}

To illustrate the performance and the effectiveness of the proposed face recognition system, the experimental results obtained from the methods explained in the above sections, and its significances are discussed. Among many of face image database that is available in world-wide, in our system, the performance of face recognition system is evaluated using ORL database. It contains a set of face images taken from 40 distinct subjects of ten different images each. All the images are taken in a dark homogeneous background where the subjects are in frontal and in an upright position. The whole database is used in order to analyze the performance of our proposed system regarding performance measure curves. The system is implemented based on the proposed system framework, and the classification results of various outputs obtained by using the different m-band filters are compared with each other and also with the Principal Component Analysis (PCA) algorithm output that which one obtains the best result.

The results of these algorithms are compared on the basis of recognition accuracy from the Receiver Operating Characteristic (ROC), Inverse ROC, Expected Performance Curve (EPC) parameters curve parameters as shown in Figure 2, 3, and 4. The performance comparisons are done between the m-band filters like HAAR 2 band, HAAR 4 band, MEYER 2 band, MEYER 4 band, SYMLET 2 band and SYMLET 4 band. These entire filter outputs are also compared with the PCA algorithm output.

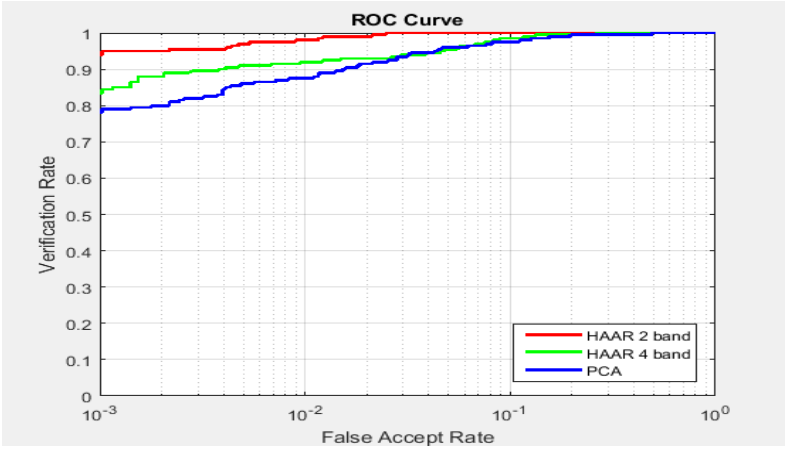

Figure 2. ROC curve for Haar 2 band vs. Haar 4 band vs. PCA

The above Figure 2 shows the ROC comparison chart between different haar m-band filters like haar 2 bands and haar 4 band filters based on the false acceptance rate and the verification rate. It is observed that the haar 2 band (RED color line) obtains the best results of $95 \%$ of accuracy when compared to the other haar 4 bands filter and PCA algorithm.

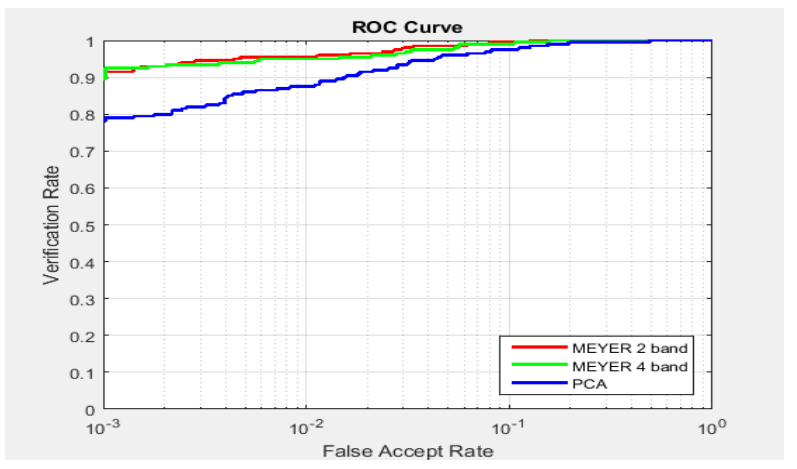

Figure 3. ROC curve for Meyer 2 band vs. Meyer 4 band vs. PCA 
Figure 3 shows the ROC comparison between different $\mathrm{m}$-band Meyer filters like Meyer 2 bands and Meyer 4 bands filter based on the false acceptance rate and the verification rate. It is observed that the Meyer 2 band (RED color line) obtains the best results of $91 \%$ of accuracy when compared to the other Meyer 4 bands filter and PCA algorithm.

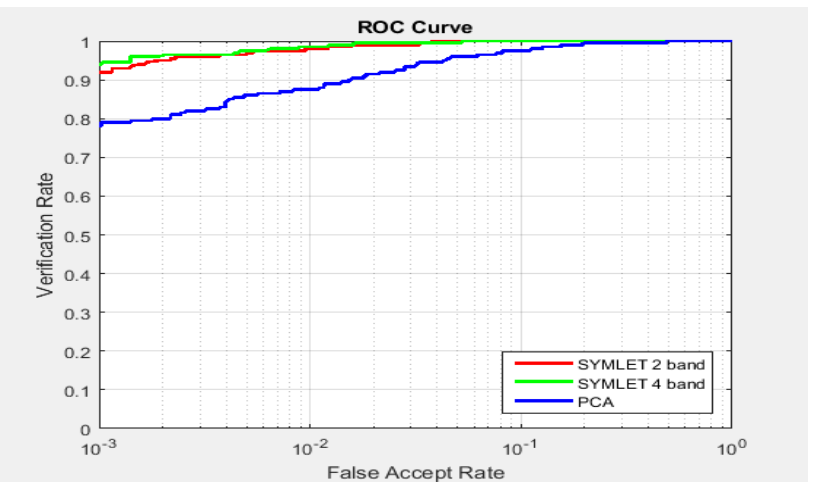

Figure 4. ROC curve for Symlet 2 band vs. Symlet 4 band vs. PCA

The above Figure 4 shows the ROC comparison between different m-band symlet filters like symlet 2 bands and symlet 4 bands filter based on the false acceptance rate and the verification rate. It is observed that the symlet 4 band (RED color line) obtains the best results of $94 \%$ of accuracy when compared to the other symlet 2 bands filter and PCA algorithm.

From the Figure 2, 3 and 4 it is observed that the best results are obtained from the filter comparisons like, in haar filters comparison, the haar 2 bands filter obtains the higher result, and between Meyer filters comparison, the Meyer 2 bands filter obtains the higher result, and in symlet filter comparison, the symlet 4 bands filter obtains the higher result.

Now the comparison is made between the three different filters with higher accuracy rates along with the PCA algorithm as shown in Figure 5, 6 and 7.

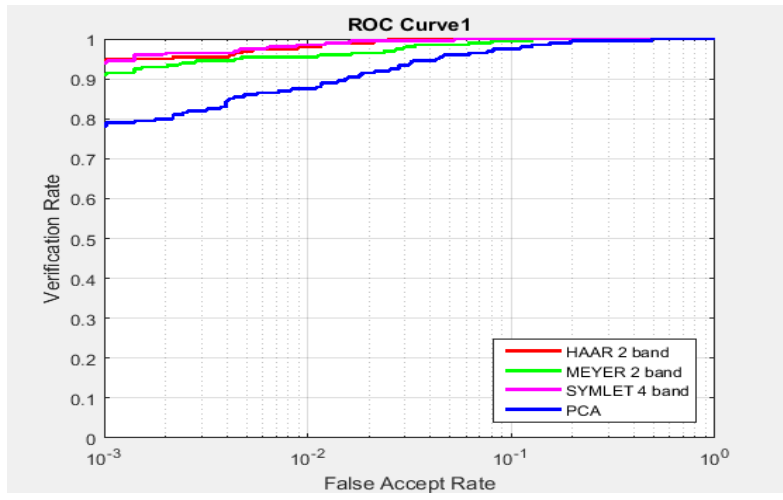

Figure 5. ROC comparison curve for three filters with best results and PCA

The ROC curve for the three different filters with best results along PCA algorithm comparison is shown in Figure 5. It is observed that among the three filters and PCA the haar 2 bands filter (RED color line) obtains the higher average accuracy rate of $95 \%$ based on false acceptance rate and the verification rate.

The inverse ROC curve for the three different filters with best results along PCA algorithm comparison based on false acceptance rate and the false rejection rate is shown in Figure 6. It is observed that among the three filters and PCA the haar 2 bands filter (RED color line) obtains the very less false rejection rate at an average ratio of $0.5 \%$ when compared to the other filters methods and PCA algorithm. 


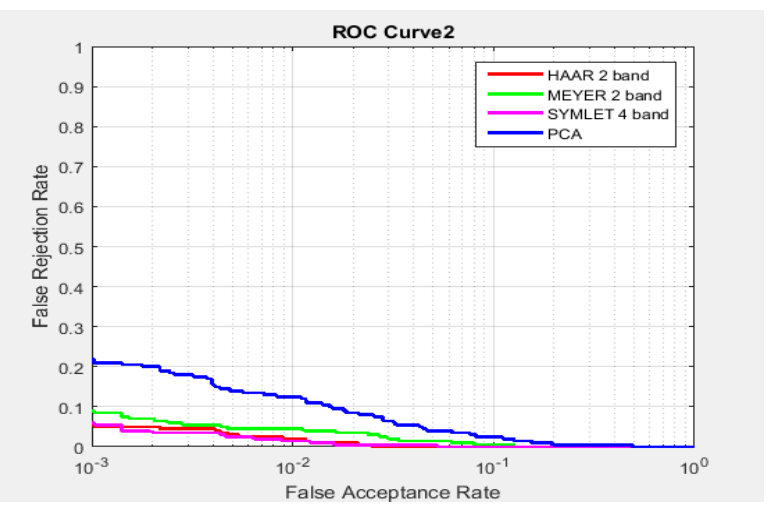

Figure 6. Inverse ROC comparison curve for three filters with best results and PCA

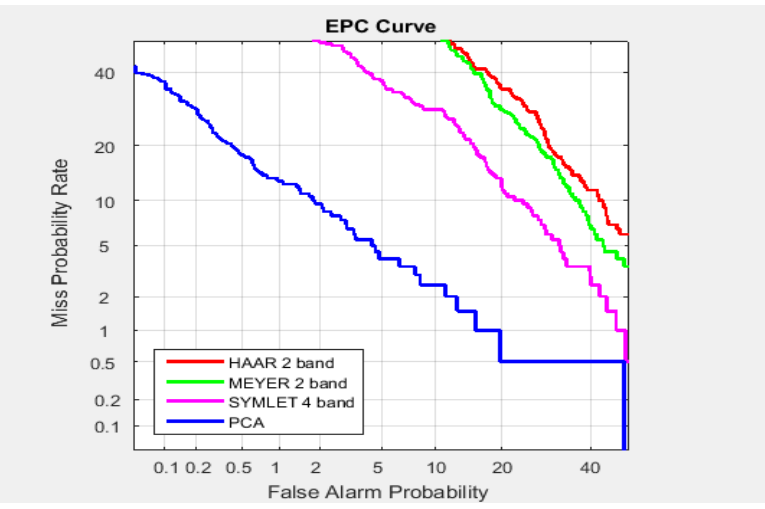

Figure 7. EPC comparison curve for three filters with best results and PCA

The above Figure 7 shows the EPC curve comparison curve for the three different filters with best results along PCA algorithm comparison based on false alarm probability rate vs miss probability rate. It is observed that among the three filters and PCA the haar 2 bands filter (RED color line) obtains the very less false missing probability rate of the classifier rate.

From the performance measure curves, it is clear that our proposed system has obtained a better performance when the features are extracted by using the m-band haar 2 bands filter. When using these parameters, the system is obtaining an average higher rate of $95 \%$ of output accuracy rates.

\section{CONCLUSION}

In our proposed work, an effective method for the person recognition system is implemented and analyzed by means of the DTMBWT with the PCA optimization and the KNN classifier algorithms. The system is implemented and worked by using the publically available face dataset known as the ORL database. Using the DTMBWT transform the sub-band coefficients are extracted in the form of features from the input images. By using the approximate sub-bands, the features are extracted from the different m-band filters like haar, Meyer and symlet. Then the classification is done by using these filters features by using the KNN classification scheme. From the results, it is proved that our proposed system produces higher accuracy results when the haar 2 band filter features. Also, the performance evolution measures are compared with the PCA algorithm and are concluded that our proposed system can produce better results and can predict the face recognition at the rate of $95 \%$ of recognition accuracy.

\section{REFERENCES}

[1] Yin X, Liu X. "Multi-task convolutional neural network for pose-invariant face recognition". IEEE Transactions on Image Processing. 2017; 1-12. 
[2] Cheng Y, Jiao L, Tong Y, Li Z, Hu Y, Cao X. "Directional illumination estimation sets and multilevel matching metric for illumination-robust face recognition". IEEE Access. 2017; 5: 25835-25845.

[3] Huang P, Gao G, Qian C, Yang G, Yang Z. "Fuzzy linear regression discriminant projection for face recognition". IEEE Access. 2017; 5: 4340-4349.

[4] Cao F, Feng X, Zhao J. "Sparse representation for robust face recognition by dictionary decomposition". Journal of Visual Communication and Image Representation. 2017; 46: 260-268.

[5] Liu W, Wen Y, Yu Z, Li M, Raj B, Song, L. "Sphereface: Deep hypersphere embedding for face recognition". IEEE Conference on Computer Vision and Pattern Recognition. 2017; 1: 1-9.

[6] Huang P, Lai Z, Gao G, Yang G, Yang Z. "Adaptive linear discriminant regression classification for face recognition". Digital Signal Processing. 2016; 55: 78-84.

[7] Masi I, Rawls S, Medioni G, Natarajan P. "Pose-aware face recognition in the wild". In Proceedings of the IEEE Conference on Computer Vision and Pattern Recognition. 2016; 4838-4846.

[8] Punnappurath A, Rajagopalan AN, Taheri S, Chellappa R, Seetharaman G. "Face recognition across non-uniform motion blur, illumination, and pose". IEEE Transactions on Image Processing. 2015; 24(7): 2067-2082.

[9] Basaran E, Gokmen M. "An efficient face recognition scheme using local Zernike moments patterns". In Asian Conference on Computer Vision. 2014; 710-724. Springer, Cham.

[10] Qian J, Luo L, Yang J, Zhang F, Lin Z. "Robust nuclear norm regularized regression for face recognition with occlusion". Pattern Recognition. 2015; 48(10): 3145-3159.

[11] Rassem TH, Makbol NM, Yee SY. "Face recognition using completed local ternary pattern texture descriptor". International Journal of Electrical and Computer Engineering(IJECE). 2017; 7(3): 1594-1604.

[12] Rahim NAA, Ghani NAM, Mohamed N, Hashim H, Musirin I. "The Application of Modified Least Trimmed Squares with Genetic Algorithms Method in Face Recognition”. Indonesian Journal of Electrical Engineering and Computer Science (IJEECS). 2017; 8(1): 154-158.

[13] El Meslouhi O, Elgarrai Z, Kardouchi M, Allali H. "Unimodal Multi-Feature Fusion and one-dimensional Hidden Markov Models for Low-Resolution Face Recognition". International Journal of Electrical and Computer Engineering (IJECE). 2017; 7(4): 1915-1922.

[14] Sable AH, Talbar SN, Dhirbasi HA. "EV-SIFT-An Extended Scale Invariant Face Recognition for Plastic Surgery Face Recognition". International Journal of Electrical and Computer Engineering (IJECE). 2017; 7(4): 1923-1933.

[15] Selesnick IW, Baraniuk RG, Kingsbury NC. "The dual-tree complex wavelet transforms". IEEE Signal Processing Magazine. 2005; 22(6): 123-151.

[16] Bhamidipati SL, Mindagudla SS, Devalla HV, Goodi HS, Nag H. "Analysis of different discrete wavelet transform basis functions in speech signal compression”. IOSR Journal of VLSI and Signal Processing, 2014; 4(1): 34-38. 